\title{
The Modified Cramer-Rao Bound for Channel Estimation in Quantize-and-Forward Cooperative Systems.
}

\author{
Iancu Avram and Marc Moeneclaey \\ Department of Telecommunications \\ and Information Processing \\ Ghent University \\ 9000 Gent, Belgium \\ Email: firstname.lastname@telin.ugent.be
}

\begin{abstract}
The quantize-and-forward (QF) cooperative protocol can effectively be used to combat fading in systems using half-duplex relay terminals. While the outage behavior of the QF protocol has been extensively investigated, few research has been performed on the impact of imperfect channel estimates. In this contribution, a lower bound (LB) on the estimation error is obtained for a one-hop relaying channel with data quantization at the relay. To this purpose the modified Cramer-Rao (MCRB) bound is calculated, which, compared to the true Cramer-Rao bound (CRB), is a looser and computationally less complex bound that converges to the CRB in the high signal-to-noise ratios. By using a general system model for the relay channel, the obtained results can be utilized to benchmark a wide variety of systems.

Index Terms-Cooperative communication, Quantize-andForward, Cramer-Rao Bound, Estimation
\end{abstract}

\section{INTRODUCTION}

Cooperative telecommunication systems can effectively be used to combat fading by exploiting the broadcast nature of the wireless medium [1], [2], [3] : a relay can forward to the destination the information it receives from the source, creating additional signal paths and thus providing spatial diversity [4], [5]. Different strategies can be used to implement the information forwarding, including amplify-and-forward (AF) [6], quantize-and-forward (QF) [7], decode-and-forward [8] and coded cooperation [9]. The AF protocol, in which the relay amplifies the signal received from the source before sending it to the destination, is well known for its seemingly low-complexity implementation. However, when half-duplex terminals are used, the information received from the source needs to be stored with high precision at the relay awaiting retransmission to the destination, requiring a large memory at the relay terminal. This memory requirement is relaxed by the quantize-and-forward $(\mathrm{QF})$ protocol, in which the signal received by the relay is (coarsely) quantized before being stored into memory. These quantized samples are then sent to the destination in a subsequent timeslot [7], [10].

The majority of the work on cooperative systems is performed under the assumption of perfect channel state information (CSI). While this is useful for obtaining various information-theoretical results, the situation of imperfect CSI presents new challenges that need to be tackled. Channel parameter estimation for QF has been discussed in [10], [11] for a protocol in which the relay estimates the source-relay (SR) channel and forwards the estimate to the destination, while [12] describes a QF system in which all channel parameters are estimated at the destination. In [12], the SR channel is abstracted to be a discrete channel, characterized by a finite set of transition probabilities. In doing so, the estimation of the SR channel is greatly simplified, and the computational complexity at the destination is reduced. In this contribution, the modified Cramer-Rao bound (MCRB) is obtained using a similar channel model as in [12]. Compaired to the unmodified Cramer-Rao bound (CRB), the MCRB is a looser and computationally less complex bound that coincides with the CRB in the high signal-to-noise (SNR) limit [13], [14]. Both the estimation of the SR channel and the relaydestination (RD) channel will be considered. By only making loose assumptions on the structure of the SR channel and the quantization operation, the results obtained here can be used to benchmark a wide variety of cooperative communication systems that use a form of quantization at the relay.

The remainder of this contribution is organized as follows. In section II the system model is introduced. In section III an expression is obtained for the modified Cramer-Rao bound (MCRB), whereafter a few special cases will be presented that will provide more insight on the qualitative aspects of the obtained lower bound (LB). Finally, the use of importance sampling (IS) is outlined, a technique that is crucial for obtaining numerical results within acceptable simulation times [16]. These numerical results are then presented in section IV, whereafter conclusions are drawn in section $\mathrm{V}$.

\section{Channel Model}

In this contribution, a one-hop relaying channel is analyzed. The relay is considered to be a half-duplex device, meaning that it cannot send and receive information simultaneously. In a first timeslot, the source broadcasts $K M_{1}$-PSK symbols, denoted $c_{s}$, which are altered by the channel before being received by the relay. The relay quantizes the received samples 
using $\log _{2}\left(M_{2}\right)$ bits. These quantized samples, represented by $\mathrm{K} \mathrm{M}_{2}$-PSK symbols denoted $\boldsymbol{c}_{r}$, are then forwarded to the destination in a second timeslot. The energy of the symbols sent by the source and the relay is equal to $E_{s}$ and $E_{r}$, respectively. By considering a memoryless SR channel and assuming that the quantization operation is performed on a symbol-by-symbol basis, the $k$-th symbol sent by the relay, denoted $c_{r}(k)$, only depends on the $k$-th symbol sent by the source, denoted $c_{s}(k)$. By noting that both the source and the relay transmit discrete symbols from a PSK constellation, the cascade of the SR channel and the quantization operation at the relay can be abstracted to be a communication channel with $M_{1}$ input values and $M_{2}$ output values. Introducing the mapping function $\chi_{M}(x)=e^{\frac{j 2 \pi x}{M}}$, the SR channel can be fully characterized by $M_{2} \times M_{1}$ transition probabilities $\tau_{q, m}(k)=$ $P\left[c_{r}(k)=\chi_{M_{2}}(q) \mid c_{s}(k)=\chi_{M_{1}}(m)\right]$, which determine the probability of the symbols sent by the relay conditioned on the symbols sent by the source.

By assuming that the duration of a frame is much shorter than the channel coherence time, the SR and RD channel parameters can be considered to be constant within a timeslot. In doing so, the transition probabilities $\tau_{q, m}(k)$ do not depend on the symbol index $k$ and will be denoted $\tau_{q, m}$. It is further assumed that $M_{2} / M_{1}$ is integer and that the quantization operation exhibits circular symmetry with respect to the symbols sent by the source, so that

$$
\begin{array}{r}
P\left[c_{r}(k)=\chi_{M_{2}}\left(q+\frac{M_{2}}{M_{1}} m\right) \mid c_{s}(k)=\chi_{M_{1}}(m)\right] \\
=P\left[c_{r}(k)=\chi_{M_{2}}(q) \mid c_{s}(k)=\chi_{M_{1}}(0)\right]
\end{array}
$$

and thus $\tau_{q+\frac{M_{2}}{M_{1} m, m}}=\tau_{q, 0}=\tau_{q}$.

The channel between the relay terminal and the destination will be modelled as a flat Rayleigh fading channel with additive white Gaussian noise. The RD channel coefficient is denoted $h_{d}$ and the samples received by the destination are represented by the vector $\boldsymbol{r}_{d}$ which is equal to

$$
\boldsymbol{r}_{d}=\sqrt{E_{r}} \boldsymbol{c}_{r} h_{d}+\boldsymbol{n}_{d} .
$$

The channel coefficient $h_{d}$ is considered to be constant during a timeslot and has a zero mean circular symmetric complex gaussian (ZMCSCG) distribution with variance $N_{h_{d}}=$ $1 / d_{R D}{ }^{n}$ with $d_{R D}$ the distance between the relay terminal and the destination and $n$ the path loss exponent. The elements of the noise vector $\boldsymbol{n}_{d}$ are ZMCSCG distributed with variance $N_{d}$.

\section{MOdified CRAMER-RAO BOUND}

The MCRB is an LB on the mean square error (MSE) of an unbiased estimate. At the destination, both the SR transition probabilities $\tau_{q}$ and the RD channel coefficient $h_{d}$ are considered to be unknown and will need to be estimated. In the current contribution, we will assume the destination does not possess any a-priori information on the different channel parameters. The unknown channel parameters are grouped into the real-valued vector $\boldsymbol{\theta}=\left(\tau_{0}, \tau_{1}, \ldots, \tau_{M_{2}-2}, \mathfrak{R}\left(h_{d}\right), \mathfrak{I}\left(h_{d}\right)\right)$, with $\mathfrak{R}\left(h_{d}\right)$ and $\mathfrak{I}\left(h_{d}\right)$ denoting the real and imaginary part of $h_{d}$, respectively. Note that $\tau_{M_{2}-1}$ is not contained in $\boldsymbol{\theta}$, because it is not an independent paremeter $\left(\tau_{M_{2}-1}=\right.$ $\left.1-\tau_{0}-\ldots-\tau_{M_{2}-2}\right)$.

In order to obtain the MCRB for the various elements of $\boldsymbol{\theta}$, the modified Fisher information matrix (MFIM) $\boldsymbol{J}_{M}(\boldsymbol{\theta})$ is introduced, the elements of which are defined as

$$
\begin{aligned}
& J_{M}(\boldsymbol{\theta})_{i, j} \\
& =\mathbb{E}_{\boldsymbol{c}_{s}}\left[\mathbb{E}_{\boldsymbol{r}_{d} \mid \boldsymbol{c}_{s}}\left[\frac{\partial}{\partial \theta_{i}} \ln p\left(\boldsymbol{r}_{\boldsymbol{d}} \mid \boldsymbol{c}_{s} ; \boldsymbol{\theta}\right) \frac{\partial}{\partial \theta_{j}} \ln p\left(\boldsymbol{r}_{\boldsymbol{d}} \mid \boldsymbol{c}_{s} ; \boldsymbol{\theta}\right)\right]\right] \\
& =\mathbb{E}_{\boldsymbol{r}_{d} \mid \boldsymbol{c}_{s}}\left[\frac{\partial}{\partial \theta_{i}} \ln p\left(\boldsymbol{r}_{\boldsymbol{d}} \mid \boldsymbol{c}_{s} ; \boldsymbol{\theta}\right) \frac{\partial}{\partial \theta_{j}} \ln p\left(\boldsymbol{r}_{\boldsymbol{d}} \mid \boldsymbol{c}_{s} ; \boldsymbol{\theta}\right)\right] .
\end{aligned}
$$

Using the notation $\hat{x}$ to represent an unbiased estimate of $x$, it follows from [13], [15] that

$$
\mathbb{E}_{\boldsymbol{r}_{d} \mid \boldsymbol{c}_{s}}\left[(\boldsymbol{\theta}-\hat{\boldsymbol{\theta}})(\boldsymbol{\theta}-\hat{\boldsymbol{\theta}})^{H}\right] \geq \boldsymbol{J}_{M}^{-1}(\boldsymbol{\theta}),
$$

where $\boldsymbol{A} \geq \boldsymbol{B}$ implies that $\boldsymbol{A}-\boldsymbol{B}$ is positive-semidefinite matrix. By noting that

$$
(\boldsymbol{\tau}-\hat{\boldsymbol{\tau}})=\boldsymbol{V}(\boldsymbol{\theta}-\hat{\boldsymbol{\theta}}),
$$

with

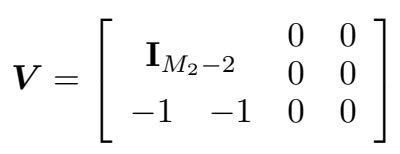

and $\mathbf{I}_{M_{2}-2}$ denoting the $\left(M_{2}-2\right) \times\left(M_{2}-2\right)$ identity matrix, a LB on the MSE arising from the estimation of $\tau$ can be expressed as

$$
\begin{aligned}
\mathbb{E}_{\boldsymbol{r}_{d} \mid \boldsymbol{c}_{s}}\left[(\boldsymbol{\tau}-\hat{\boldsymbol{\tau}})^{H}(\boldsymbol{\tau}-\hat{\boldsymbol{\tau}})\right]=\operatorname{Tr}\left(\mathbb{E}_{\boldsymbol{r}_{d} \mid \boldsymbol{c}_{s}}\left[(\boldsymbol{\tau}-\hat{\boldsymbol{\tau}})(\boldsymbol{\tau}-\hat{\boldsymbol{\tau}})^{H}\right]\right) \\
=\operatorname{Tr}\left(\boldsymbol{V} \mathbb{E}_{\boldsymbol{r}_{d} \mid \boldsymbol{c}_{s}}\left[(\boldsymbol{\theta}-\hat{\boldsymbol{\theta}})(\boldsymbol{\theta}-\hat{\boldsymbol{\theta}})^{H}\right] \boldsymbol{V}^{H}\right) \\
\geq \operatorname{Tr}\left(\boldsymbol{V} \boldsymbol{J}_{M}^{-1}(\boldsymbol{\theta}) \boldsymbol{V}^{H}\right)=\operatorname{MCRB}_{\tau}(\boldsymbol{\theta}),
\end{aligned}
$$

where in the last step (4) was used. Similarly, $\left(h_{d}-\hat{h_{d}}\right)$ can be expressed as

$$
\left(h_{d}-\hat{h_{d}}\right)=\boldsymbol{u}(\boldsymbol{\theta}-\hat{\boldsymbol{\theta}}),
$$

with $\boldsymbol{u}=(0, \ldots, 0,1, i)$, yielding the following LB on the MSE arising from the estimation of $h_{d}$ :

$$
\begin{aligned}
\mathbb{E}_{\boldsymbol{r}_{d} \mid \boldsymbol{c}_{s}}\left[\left|h_{d}-\hat{h_{d}}\right|^{2}\right] & =\operatorname{Tr}\left(\boldsymbol{u} \mathbb{E}\left[(\boldsymbol{\theta}-\hat{\boldsymbol{\theta}})(\boldsymbol{\theta}-\hat{\boldsymbol{\theta}})^{H}\right] \boldsymbol{u}^{H}\right) \\
& \geq \operatorname{Tr}\left(\boldsymbol{u} \boldsymbol{J}_{M}^{-1}(\boldsymbol{\theta}) \boldsymbol{u}^{H}\right)=\operatorname{MCRB}_{h_{d}}(\boldsymbol{\theta}) .
\end{aligned}
$$

Note that the exact value of the MCRB from (6) and (8) depends on the specific realization of $\boldsymbol{\theta}$. In order to obtain a LB that is independent of $\boldsymbol{\theta}$, (6) and (8) need to be averaged over $\boldsymbol{\theta}$, as will be done numerically in section IV. It is shown in Appendix A that for a given value of $\boldsymbol{\theta}$, the elements of the MFIM can be expressed as

$$
J_{M}(\boldsymbol{\theta})_{i, j}=K \mathbb{E}_{r_{d} \mid c_{s}}\left[X_{i, j}\left(r_{d}, c_{s} ; \boldsymbol{\theta}\right)\right],
$$

where $c_{s}$ is an arbitrary $M_{1}$-PSK constellation point, and the expectation is over the distribution $p\left(r_{d} \mid c_{s}\right)$, representing 
a short-hand notation of $p\left(r_{d}(k) \mid c_{s}(k)=c_{s}\right)$, which does not depend on the time index $k$. The exact expression for $X_{i, j}\left(\boldsymbol{r}_{d}, \boldsymbol{c}_{s} ; \boldsymbol{\theta}\right)$ is given in (25).

\section{A. Special Cases}

In this subsection, a few special cases are introduced which are simpler to evaluate than the general case, and which provide a lower bound on the MCRB resulting from (9).

1) Perfect RD channel: A perfect RD channel corresponds to the case where the destination receives the message from the relay unaltered, i.e., $\boldsymbol{r}_{d}=\boldsymbol{c}_{r}$. When this is the case, only the parameters $\left\{\theta_{q}, q=0, \ldots, M_{2}-2\right\}$ must be estimated. The MFIM has dimension $\left(M_{2}-1\right) \times\left(M_{2}-1\right)$, and is obtained by substituting in (25) $\boldsymbol{c}_{r}$ for $\boldsymbol{r}_{d}$, yielding

$$
\begin{aligned}
& \boldsymbol{J}_{M}(\boldsymbol{\theta})_{i, j} \\
& \quad=K \mathbb{E}_{c_{r} \mid c_{s}}\left[\frac{\partial}{\partial \theta_{i}} \ln p\left(c_{r} \mid c_{s} ; \boldsymbol{\theta}\right) \frac{\partial}{\partial \theta_{j}} \ln p\left(r_{d} \mid c_{s} ; \boldsymbol{\theta}\right)\right] .
\end{aligned}
$$

Using (26), (10) can be written as

$$
\boldsymbol{J}_{M}(\boldsymbol{\theta})_{i, j}=\frac{\delta_{i-j}}{\tau_{i}}+\frac{1}{\tau_{M_{2}-1}},
$$

with $\delta_{x}=1$ only if $x=0$, yielding the following expression for the MFIM:

$$
\boldsymbol{J}_{M}(\boldsymbol{\theta})=\boldsymbol{D}^{-1}+\tau_{M_{2}-1}^{-1} \mathbf{1 1 ^ { T }},
$$

with $\boldsymbol{D}$ a diagonal matrix with diagonal elements equal to $\left(\tau_{0}, \tau_{1}, \ldots, \tau_{M_{2}-2}\right)$ and $\mathbf{1}$ a column vector with all elements equal to 1 . Introducing $\boldsymbol{d}=\left(\tau_{0}, \tau_{1}, \ldots, \tau_{M_{2}-2}\right)^{T}$ and using the matrix inversion lemma to calculate the inverse of the MFIM yields

$$
\boldsymbol{J}_{M}^{-1}(\boldsymbol{\theta})=\frac{1}{K}\left(\boldsymbol{D}-\boldsymbol{d} \boldsymbol{d}^{T}\right) .
$$

The MCRB corresponding to the estimation of $\tau$ is obtained by substituting (13) into (6), yielding

$$
\mathbb{E}\left[(\boldsymbol{\tau}-\hat{\boldsymbol{\tau}})^{H}(\boldsymbol{\tau}-\hat{\boldsymbol{\tau}})\right] \geq \frac{1}{K}\left(1-\sum_{q=0}^{M_{2}-1} \tau_{q}^{2}\right) .
$$

2) Perfect SR channel: A perfect SR channel corresponds to the case where the relay receives the message from the source unaltered; the message sent by the relay then equals a quantized version of the message sent by the source. This yields $\boldsymbol{c}_{r}=F_{Q}\left(\sqrt{E_{s}} \boldsymbol{c}_{s}\right)$, with $F_{Q}($.$) representing the quan-$ tization operation. In this case we only have to estimate $h_{d}$. The corresponding MFIM of dimension $2 \times 2$ is obtained by substituting in (25) $\boldsymbol{c}_{r}$ for $\boldsymbol{c}_{s}$. One obtains $\boldsymbol{J}_{M}=\left(\frac{2 K E_{r}}{N_{d}}\right) \mathbf{I}_{2}$, and thus

$$
\mathbb{E}\left[\left|\hat{h_{d}}-h_{d}\right|^{2}\right] \geq \frac{N_{d}}{K E_{r}} .
$$

3) Known $S R$ or RD channel: When the transition probabilities of the SR channel are known, the MCRB corresponding to the estimation of the RD channel $h_{d}$ is obtained by omitting from the MFIM the first $M_{2}-1$ columns and rows that correspond to the parameters $\theta_{0}, \theta_{1}, \ldots, \theta_{M_{2}-2}$ of the SR channel. Similarly, when the RD channel $h_{d}$ is known, the MFIM for the estimation of the SR channel parameters $\theta_{0}, \theta_{1}, \ldots, \theta_{M_{2}-2}$ is obtained by removing from the MFIM the last two rows and columns.

\section{B. Importance Sampling}

Except in the case of a perfect RD or SR channel, averaging over $\boldsymbol{r}_{d}$ in (9) cannot be achieved analytically and MonteCarlo (MC) techniques must be employed. However, when some of the transition probabilities of the SR channel are very small, the computational effort required to achieve a consistent value for the MCRB can be prohibitively high. This can be understood by calculating the MCRB corresponding to the case of a perfect RD channel and using a $\mathrm{MC}$ approach instead of an analytical one. The MC approach to obtain $J_{M}(\boldsymbol{\theta})_{i, j}$ involves the following approximation :

$$
\begin{aligned}
J_{M}(\boldsymbol{\theta})_{i, j} \approx \frac{K}{N} \sum_{n=1}^{N} \frac{I_{i}^{(n)} \delta_{i-j}}{\tau_{i}^{2}}+ & \frac{I_{M_{2}-1}^{(n)}}{\tau_{M_{2}-1}^{2}} \\
& i, j=0,1, \ldots, M_{2}-2,
\end{aligned}
$$

where $I_{q}^{(n)}=1$ if $c_{r}^{(n)}=c_{s} e^{\frac{j 2 \pi q}{M_{2}}}$ and 0 otherwise, $c_{s}$ is an arbitrary symbol from the $M_{1}$-PSK constellation (say, $c_{s}=1$ ), and $\left\{c_{r}^{(n)}, n=1,2, \ldots, N\right\}$ is a sequence of i.i.d random variables, generated according to $\operatorname{Pr}\left[c_{r}^{(n)}=c_{s} e^{\frac{j 2 \pi q}{M_{2}}}\right]=\tau_{q}, q=$ $0,1, \ldots, M_{2}-1$. Suppose that for given $\mathrm{N}, \tau_{i 1}$ and $\tau_{i 2}$ are both much smaller than $1 / N$, so that it is likely to have $I_{i 1}^{(n)}=I_{i 2}^{(n)}=0$ for $n=1,2, \ldots, N$. In this case, the (estimate of) the MFIM obtained from (16) is singular, so that its inverse does not exist. Hence, to obtain meaningful results using (16), very large values of $\mathrm{N}$ (and, therefore, long simulation times) are required when some of the transition probabilities are very small.

This problem can be avoided by using importance sampling (IS) [16]. For the situation at hand, this involves generating $\left\{c_{r}^{(n)}, n=1,2, \ldots, N\right\}$ according to a biased distribution $\operatorname{Pr}\left[c_{r}^{(n)}=c_{s} e^{\frac{j 2 \pi q}{M_{2}}}\right]=\kappa_{q}, q=0,1, \ldots, M_{2}-1$, and replacing (16) by

$$
\begin{aligned}
J_{M}(\boldsymbol{\theta})_{i, j} \approx \frac{K}{N} \sum_{n=1}^{N} \frac{I_{i}^{(n)} \delta_{i-j}}{\tau_{i}^{2}} \cdot \frac{\tau_{i}}{\kappa_{i}} & +\frac{I_{M_{2}-1}^{(n)}}{\tau_{M_{2}-1}^{2}} \cdot \frac{\tau_{M_{2}-1}}{\kappa_{M_{2}-1}} \\
i, j & =0,1, \ldots, M_{2}-2 .
\end{aligned}
$$

We select all $\kappa_{q}$ to be much larger than $1 / N$, so that it is unlikely for any $q$ to have $I_{q}^{(n)}=0$ for $n=1,2, \ldots, N$. This yields $\frac{1}{N} \sum_{n=1}^{N} I_{q}^{(n)} \approx \kappa_{q}$ for all $q$, so that (17) is close to the analytical result (11). 
In the general case, $J_{M}(\boldsymbol{\theta})$ from (9) is computed as

$$
J_{M}(\boldsymbol{\theta})_{i, j} \approx \frac{K}{N} \sum_{n=1}^{N} X_{i, j}\left(r_{d}, c_{s} ; \boldsymbol{\theta}\right) \cdot \frac{p\left(r_{d}^{(n)} \mid c_{s} ; \boldsymbol{\theta}\right)}{p\left(r_{d}^{(n)} \mid c_{s} ; \tilde{\boldsymbol{\theta}}\right)},
$$

where $\left\{r_{d}^{(n)}, n=1,2, \ldots, N\right\}$ are $\mathrm{N}$ independent realizations generated according to the biased distribution $p\left(r_{d}^{(n)} \mid c_{s} ; \tilde{\boldsymbol{\theta}}\right)$.

\section{NUMERICAL RESULTS}

In this section, the value of the MCRB is obtained for different SNR ratios using computer simulations. Before results can be obtained, the SR channel and the quantization operation need to be parameterized. To this purpose, the SR channel is modelled as a flat Rayleigh fading channel with additive white Gaussian noise, characterized by the channel parameter $h_{r}$. The latter is constant during a timeslot and has a ZMCSCG distribution with variance $N_{h_{r}}=1 / d_{S R}{ }^{n}$, with $d_{S R}$ the distance between the source and the relay terminal. The additive white Gaussian noise has a ZMCSCG distribution with variance $N_{r}$. The path-loss exponent on the SR and RD channels is assumed to be equal to 4 and the distances between the various terminals satisfy $d_{S R}=d_{R D}=0.5$. The SR and RD channels have equal noise variances (i.e. $N_{r}=N_{d}$ ) and equal symbol energies (i.e. $E_{s}=E_{r}$ ). The source is assumed to broadcast BPSK symbols that are quantized at the relay on QPSK symbols. The quantization of the samples at the relay is performed without knowledge of the SR channel as described in [7], [12].

The corresponding transition probabilities are obtained as function of the SR channel parameters $h_{r}$ and $E_{s} / N_{r}$ using the techniques described in [7], [12]. Because some transition probabilities can be very small at high SNR values, the use of IS is required for obtaining meaningfull results within reasonable simulation times. The alternative transition probabilities $\kappa$ used for the IS distribution are generated by assuming the instantaneous SNR of the SR channel, defined as $\gamma_{r}=E_{s}\left|h_{r}\right|^{2} / N_{r}$ is always equal to $0 \mathrm{~dB}$, independent of the actual value. In doing so, only $10^{4}$ realizations of $r_{d}$ given $c_{s}$ are required to average over $r_{d}$ in (9). The framelength $K$ is equal to 1500 and a total of $10^{4}$ realizations of $\boldsymbol{\theta}$ are used to average $\operatorname{MCRB}_{\tau}(\boldsymbol{\theta})$ and $\operatorname{MCRB}_{h_{d}}(\boldsymbol{\theta})$ over $\boldsymbol{\theta}$.

Fig. 1 shows the MCRB corresponding to the estimation of the SR channel as function of the average SNR on this channel (defined as $\mathrm{SNR}_{r}=\mathbb{E}\left[\gamma_{r}\right]=E_{s} N_{h_{r}} / N_{r}$ ) for various values of the instantaneous SNR on the RD channel (defined as $\gamma_{d}=E_{r}\left|h_{d}\right|^{2} / N_{d}$ ). Also included for reference is the case of a perfectly known RD channel, corresponding to the case of $\gamma_{d} \rightarrow+\infty$. As can be seen on the plot, $\mathrm{MCRB}_{\tau}$ decreases as $\mathrm{SNR}_{r}$ increases. It is worth to note however that the value of $\mathrm{MCRB}_{\tau}$ is much more sensitive to the value of $\gamma_{d}$. When $\gamma_{d}$ decreases, the a posteriori probabilities $p\left(c_{r} \mid r_{d}, c_{s} ; \boldsymbol{\theta}\right)$ tend to $p\left(c_{r} \mid c_{s} ; \boldsymbol{\theta}\right)$; in which case the elements (26) of the MFIM related to the estimation of the transition probabilities become very small, and the corresponding MCRB gets very large. When $\gamma_{d}$ is very large, $p\left(c_{r} \mid r_{d}, c_{s} ; \boldsymbol{\theta}\right) \approx 1$ when $c_{r}$ is the

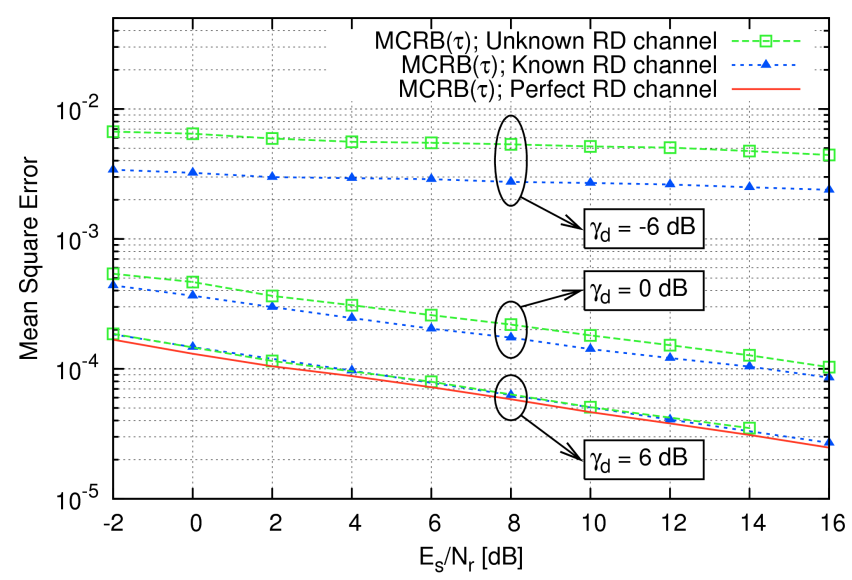

Fig. 1. MSE of $\hat{\tau}$ as a function of the $E_{s} / N_{r}$ ratio for different states of the RD channel.

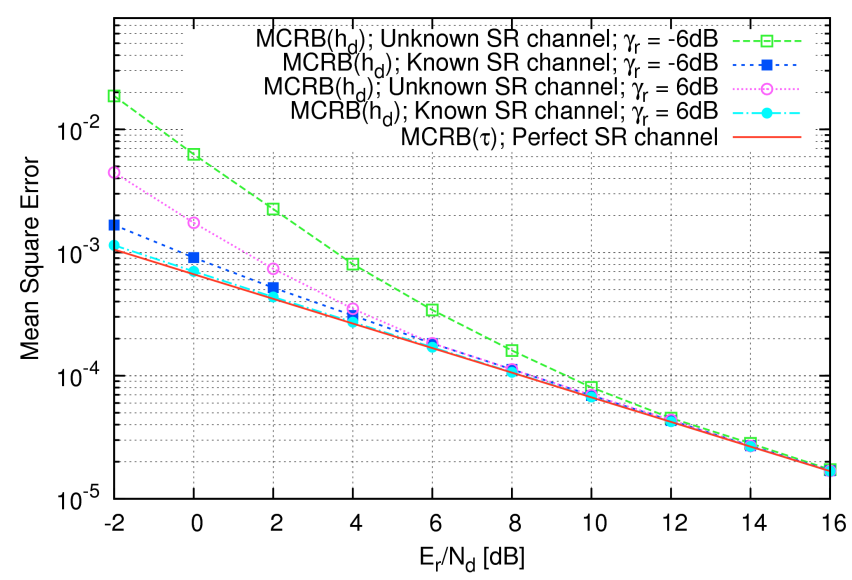

Fig. 2. MSE of $\hat{h_{2}}$ as a function of the $E_{r} / N_{d}$ ratio for different states of the SR channel.

$M_{2}$-PSK constellation point actually transmitted by the relay; the corresponding MCRB related to estimating $\tau$ approaches the MCRB (14) for the perfect RD channel, which assumes a maximum value of $\left(1-\frac{1}{M_{2}}\right) / K$ when $\mathrm{SNR}_{r}$ goes to zero, and approaches zero when $\mathrm{SNR}_{r}$ goes to infinity.

The MCRB related to the estimation of the RD channel is shown in Fig. 2 as function of the average SNR on this channel (defined as $\mathrm{SNR}_{d}=\mathbb{E}\left[\gamma_{d}\right]=E_{r} N_{h_{d}} / N_{d}$ ), for different values of $\gamma_{r}$. The situation of a perfect SR channel is also included for reference. Unlike the MCRB related to the estimation of the SR channel transition probabilities, the MCRB related to the estimation of $h_{d}$ is much less sensitive to variations in the instantaneous SNR of the SR channel, especially at high SNR values. This is due to the fact that the relay broadcasts discrete symbols from a pre-defined and known constellation, information which can be used when estimating the RD channel coefficient. When $\mathrm{SNR}_{d}$ is very large, $p\left(c_{r} \mid r_{d}, c_{s} ; \boldsymbol{\theta}\right) \approx 1$ when $c_{r}$ is the $M_{2}$-PSK constellation point actually transmitted by the relay; from (27) it can be 
verified that in this case $\mathrm{MCRB}_{h_{d}}$ approaches the MCRB (15) for the perfect SR channel. This implies that at high SNR, the symbol sent by the relay can be accurately determined without the knowledge of the symbol sent by the source, as the distance between the constellation points at the relay becomes larger compared to the noise variance. As a result, from an estimation point of view, the optimal relay location is close to the destination, as this has the best potential to yield accurate channel estimates for both the SR and RD channels.

\section{CONCLUSIONS}

In this contribution, LBs were derived for the estimation of the SR channel and the RD channel in QF cooperative systems. The SR channel and the quantization operation only need to adhere to loose constraints outlined in section II, making the obtained results applicable to a wide variety of cases, such as Rice fading channels or a cascade of different channels. After presenting an expression for the MCRB, a few special cases were introduced in which the MCRB can be obtained analytically. In the general case, the value of the MCRB is obtained using IS techniques. The presented results show that it is difficult to obtain an accurate estimate of the SR channel parameters when the RD channel quality is low. Because a low SNR on the SR channel does not significantly deteriorate the $\mathrm{RD}$ channel estimate, placing the relay close to the destination has the best potential to yield accurate channel estimates for both the SR and RD channel. Future work includes extending the obtained expressions to general QAM constellations.

\section{APPENDIX A \\ CALCULATION OF THE MODIFIED FISHER INFORMATION MATRIX}

In the following section, the calculation of the elements of the modified Fisher information matrix is outlined. These elements are obtained by evaluating

$$
\begin{aligned}
\boldsymbol{J}_{M}(\boldsymbol{\theta})_{i, j} & =\mathbb{E}_{\boldsymbol{r}_{d} \mid \boldsymbol{c}_{s}}\left[\frac{\partial}{\partial \theta_{i}} \ln p\left(\boldsymbol{r}_{d} \mid \boldsymbol{c}_{s} ; \boldsymbol{\theta}\right) \frac{\partial}{\partial \theta_{j}} \ln p\left(\boldsymbol{r}_{d} \mid \boldsymbol{c}_{s} ; \boldsymbol{\theta}\right)\right] \\
& =\mathbb{E}_{\boldsymbol{r}_{d} \mid \boldsymbol{c}_{s}}\left[g_{i}\left(\boldsymbol{r}_{d}, \boldsymbol{c}_{s} ; \boldsymbol{\theta}\right) g_{j}\left(\boldsymbol{r}_{d}, \boldsymbol{c}_{s} ; \boldsymbol{\theta}\right)\right],
\end{aligned}
$$

with $g_{i}\left(\boldsymbol{r}_{d}, \boldsymbol{c}_{s} ; \boldsymbol{\theta}\right)=\frac{\partial}{\partial \theta_{i}} \ln p\left(\boldsymbol{r}_{d} \mid \boldsymbol{c}_{s} ; \boldsymbol{\theta}\right)$. By taking into consideration the channel model described in section II, the function $g_{i}$ can be written as

$$
\begin{aligned}
g_{i}\left(\boldsymbol{r}_{d}, \boldsymbol{c}_{s} ; \boldsymbol{\theta}\right) & =\frac{\partial}{\partial \theta_{i}} \ln \prod_{k=1}^{K} p\left(r_{d}(k) \mid c_{s}(k) ; \boldsymbol{\theta}\right) \\
& =\frac{\partial}{\partial \theta_{i}} \sum_{k=1}^{K} \ln p\left(r_{d}(k) \mid c_{s}(k) ; \boldsymbol{\theta}\right)
\end{aligned}
$$

with

$$
\begin{array}{r}
\ln p\left(r_{d}(k) \mid c_{s}(k) ; \boldsymbol{\theta}\right)= \\
\sum_{c_{r}(k)} p\left(r_{d}(k) \mid c_{r}(k) ; \boldsymbol{\theta}\right) p\left(c_{r}(k) \mid c_{s}(k) ; \boldsymbol{\theta}\right),
\end{array}
$$

where the summation over $c_{r}(k)$ runs over all $M_{2}$ values $c_{r}(k)$ can adopt. Substituting (20) into (19) yields

$$
\begin{aligned}
& \boldsymbol{J}_{M}(\boldsymbol{\theta})_{i, j}=\mathbb{E}_{\boldsymbol{r}_{d} \mid \boldsymbol{c}_{s}} \\
& {\left[\sum_{k, \tilde{k}=1}^{K} \frac{\partial}{\partial \theta_{i}} \ln p\left(r_{d}(k) \mid c_{s}(k) ; \boldsymbol{\theta}\right) \frac{\partial}{\partial \theta_{j}} \ln p\left(r_{d}(\tilde{k}) \mid c_{s}(\tilde{k}) ; \boldsymbol{\theta}\right)\right] .}
\end{aligned}
$$

Evaluation of the terms for which $k \neq \tilde{k}$ yields

$$
\begin{gathered}
\mathbb{E}_{\boldsymbol{r}_{d} \mid \boldsymbol{c}_{s}}\left[\frac{\partial}{\partial \theta_{i}} \ln p\left(r_{d}(k) \mid c_{s}(k) ; \boldsymbol{\theta}\right) \frac{\partial}{\partial \theta_{j}} \ln p\left(r_{d}(\tilde{k}) \mid c_{s}(\tilde{k}) ; \boldsymbol{\theta}\right)\right] \\
=\mathbb{E}_{r_{d}(k) \mid c_{s}(k)}\left[\frac{\partial}{\partial \theta_{i}} \ln p\left(r_{d}(k) \mid c_{s}(k) ; \boldsymbol{\theta}\right)\right] \\
. \mathbb{E}_{r_{d}(\tilde{k}) \mid c_{s}(\tilde{k})}\left[\frac{\partial}{\partial \theta_{j}} \ln p\left(r_{d}(\tilde{k}) \mid c_{s}(\tilde{k}) ; \boldsymbol{\theta}\right)\right], \quad(23)
\end{gathered}
$$

with

$$
\begin{aligned}
\mathbb{E}_{r_{d}(k) \mid c_{s}(k)} & {\left[\frac{\partial}{\partial \theta_{i}} \ln p\left(r_{d}(k) \mid c_{s}(k) ; \boldsymbol{\theta}\right)\right] } \\
& =\int \frac{\partial}{\partial \theta_{i}} p\left(r_{d}(k) \mid c_{s}(k) ; \boldsymbol{\theta}\right) \mathrm{d} r_{d}(k)=0 .
\end{aligned}
$$

Substituting (23), (24) into (22) yields

$$
\begin{aligned}
& \boldsymbol{J}_{M}(\boldsymbol{\theta})_{i, j}=\sum_{k=1}^{K} \mathbb{E}_{r_{d}(k) \mid c_{s}(k)} \\
& {\left[\frac{\partial}{\partial \theta_{i}} \ln p\left(r_{d}(k) \mid c_{s}(k) ; \boldsymbol{\theta}\right) \frac{\partial}{\partial \theta_{j}} \ln p\left(r_{d}(k) \mid c_{s}(k) ; \boldsymbol{\theta}\right)\right] } \\
&= K \mathbb{E}_{r_{d}(0) \mid c_{s}(0)} \\
& {\left[\frac{\partial}{\partial \theta_{i}} \ln p\left(r_{d}(0) \mid c_{s}(0) ; \boldsymbol{\theta}\right) \frac{\partial}{\partial \theta_{j}} \ln p\left(r_{d}(0) \mid c_{s}(0) ; \boldsymbol{\theta}\right)\right] . }
\end{aligned}
$$

The factors $\frac{\partial}{\partial \theta_{i}} \ln p\left(r_{d}(0) \mid c_{s}(0) ; \boldsymbol{\theta}\right)$ in (25) can further be expressed as

$$
\begin{aligned}
& \frac{\partial}{\partial \theta_{i}} \ln p\left(r_{d} \mid c_{s} ; \boldsymbol{\theta}\right) \\
& =\frac{P\left[c_{r}=\chi_{M_{2}}\left(i+\frac{M_{2}}{M_{1}} m\right) \mid r_{d}, c_{s}=\chi_{M_{1}}(m) ; \boldsymbol{\theta}\right]}{\tau_{i}} \\
& -\frac{P\left[c_{r}=\chi_{M_{2}}\left(\left(M_{2}-1\right)+\frac{M_{2}}{M_{1}} m\right) \mid r_{d}, c_{s}=\chi_{M_{1}}(m) ; \boldsymbol{\theta}\right]}{\tau_{M_{2}-1}}
\end{aligned}
$$

for $i<M_{2}-1$ and

$$
\frac{\partial}{\partial \theta_{i}} \ln p\left(r_{d} \mid c_{s} ; \boldsymbol{\theta}\right)=\sum_{c_{r}} \frac{\partial}{\partial \theta_{i}} \ln p\left(r_{d} \mid c_{r} ; \boldsymbol{\theta}\right) p\left(c_{r} \mid c_{s}, r_{d} ; \boldsymbol{\theta}\right)
$$

for $i \geq M_{2}-1$, where the symbol index 0 has been omitted for convenience of notation.

\section{ACKNOWLEDGMENT}

The authors wish to acknowledge the Agency for Innovation by Science and Technology Flanders (IWT) that motivated this work. 


\section{REFERENCES}

[1] B. Sklar Rayleigh Fading Channels in Mobile Digital Communication Systems Part I: Characterization. IEEE Communications Magazine, vol. 35, no. 7, pp. 90-100, Jul. 1997

[2] T. M. Cover and J. A. Thomas Elements of Information Theory. Wiley, New York, 1991

[3] A. Sendonaris, E. Erkip and B. Aazhang User cooperation diversity part I:system Description. IEEE Trans. on Commun., vol. 51, no. 11, pp. 1927-1938, Nov. 2003

[4] T. M. Cover and A. El Gamal Capacity theorems for the relay channel. IEEE Trans. Inf. Theory, vol. 25, no. 5, pp. 572-584, Sep. 1979

[5] A. El Gamal, M. Mohseni and S. Zahedi Bounds on capacity and minimum energy-per-bit for AWGN relay channels. IEEE Trans. Inf. Theory, vol. 52, no. 4, pp. 1545-1561, Apr. 2006

[6] J. N. Laneman Cooperative Diversity in Wireless networks: Algorithms and Architectures. Massachusetts Institute of Technology, 2002

[7] M. Souryal and H. You Quantize-and-Forward Relaying with M-ary Phase Shift Keying. IEEE Wireless Communications and Networking Conference 2008, pp. 42-47

[8] J. N. Laneman, D. N. C. Tse and G. W. Wornell Cooperative diversity in wireless networks: efficient protocols and outage behavior. IEEE Trans. Inf. Theory., vol. 50, no. 12, pp. 3062-3080, Dec. 2004

[9] T. E. Hunter, S. Sanayei and A. Nosratinia Outage Analysis of Coded Cooperation. IEEE Trans. Inf. Theory., vol. 52, no. 2, pp. 375-391, Feb. 2006

[10] I. Avram, N. Aerts, D. Duyck, and M. Moeneclaey A Novel Quantizeand-Forward Cooperative System: Channel Estimation and M-PSK Detection Performance. EURASIP Journal on Wireless Communications and Networking, vol. 2010, Article ID 415438, 11 pages

[11] I. Avram, N. Aerts and M. Moeneclaey A Novel Quantize-and-Forward Cooperative System: Channel Parameter Estimation Techniques. Future Network \& Mobile Summit 2010, Florence, Italy, 16-18 Jun. 2010

[12] I. Avram, N. Aerts, H. Bruneel and M. Moeneclaey Quantize and Forward Cooperative Communication: Channel Parameter Estimation. IEEE Trans. on Wireless Commun., Vol. 11, no. 3, pp. 1167-1179, March 2012.

[13] A. D. Andrea, U. Mengali, and R. Reggiannini The modified CramerRao bound and its application to synchronization problems. IEEE Trans. Commun., vol. 42, pp. 1391-1399, Feb.-Apr. 1994.

[14] M. Moeneclaey On the True and the Modified Cramer-Rao Bounds for the Estimation of a Scalar Parameter in the Presence of Nuisance Parameters. IEEE Trans. Commun., vol. 46, no. 11, pp. 1536-1544, Nov. 1998.

[15] C. R. Rao Information and the Accuracy Attainable in the Estimation of Statistical Parameters. Bulletin of Cal. Math. Soc., vol. 37, no. 3, pp. 81-91, 1945

[16] J. M. Hammersley and D. C. Handscomb Monte Carlo Methods. Methuen \& Co., London, and John Wiley \& Sons, New York, 1964 